\title{
A Proposal of Clinical Report Regarding Dynamic Emg in Gait Disorders Following Upper Motoneuron Lesion
}

\author{
Mario Manca* and Pia Marchi
}

\author{
Laboratory of Movement Analysis, Department of Neuroscience and Rehabilitation Medicine, Azienda Ospedaliero, \\ Universitaria di Ferrara, Italy
}

\begin{abstract}
A report which includes patient problems, descriptions of clinical and instrumental assessment and conclusion with suggestions for decision making, is a necessary tool for an adequate approach to the patient.

In this paper we tried to define those steps we believe to be useful to produce a clinical report for patients with ambulation disability submitted to dynamic gait emg: Brief description of the patient's history; Description of the deformities; Observational gait analysis; Report of the items relevant to the deformities and/or relevant for treatment proposals; List of recorded muscles; Emg timing pattern; Conclusions leading to treatment proposals.
\end{abstract}

The emg timing pattern can be useful for the identification of peripheral and central impairments (contracture, paretic, spastic, co-contraction components), which can significantly interfere with the gait profile of the single patient.

We can use forms and tables which include emg signals features and recording parameters, physical examination and observational gait analysis notes. Attention must be paid for an adequate support of literature.

Keywords: Clinical report, dynamic EMG, upper motoneuron lesions, gait.

Patients with upper motoneuron (UM) lesions frequently present impairments in lower limb function which influence normal walking. Gait rehabilitation is an important goal because the ability to walk significantly influences the patients' chances of successfully returning to their premorbid environment. To improve the gait of the patients, therapists and rehabilitation teams attempt to improve the various impairments of hemiparetic subjects, such as paretic lower limb muscle strength, balance, motor function, sensation, and muscle tone. Consequently, it is important to understand the characteristics and underlying motor control of gait in patients with UM lesions.

Human gait is conventionally described in terms of "gait cycle"(GC) and represents a sequence of events between two sequential initial contacts with the floor by the same limb. The gait cycle is divided into two principal periods: stance and swing. The stance is usually considered the first period of $\mathrm{GC}$ and corresponds to when the foot is on the ground for the entire period and lasts for approximately the first $60 \%$ of GC time, the swing period begins as the foot is lifted from the floor and continues for the last $40 \%$ of the cycle. J. Perry [1] subdivided gait cycle and the stance and swing periods into three functional tasks; each task includes some phases: 1) During the Weight Acceptance (10\% of the GC) the challenge is the transfer of body weight onto a limb that has just finished swinging and has an unstable alignment. It includes two phases: Initial Contact (IC) which is the moment when the foot lands on the ground (the way the foot makes contact will influence the limb's loading) and

*Address correspondence to this author at the Laboratory of Movement Analysis, Department of Neuroscience and Rehabilitation Medicine, Azienda Ospedaliero, Universitaria di Ferrara, Italy; Tel: + 390532 238719; Fax:+390532 238703; E-mail: m.manca@ospfe.it
Loading Response (LR) when the body decelerates, in this phase the shock of impact is absorbed, weight-bearing stability is distributed over two limbs, progression is preserved. 2) At the Single Limb Support (40\% of the GC) the limb has the total responsibility for supporting body weight and can be subdivided into Mid and Terminal Stance phases. Mid Stance (MSt) begins as the other foot is lifted until the Center of Mass (CoM) is aligned over the forefoot, and implies the progression over the stationary foot and limb and trunk stability; at the Terminal Stance (TSt) the CoM moves ahead of the forefoot producing a body acceleration beyond the supporting foot. 3) The Limb Advancement (50\% of the GC) begins in stance with preparatory_posturing then it continues in swing and prepares the next contact with the floor. It includes four phases: Pre Swing (PSw), the final phase of Stance and second phase of double support, the limb prepares for swing by the weight transfer over the other limb; Initial Swing (ISw), the first phase of swing, the foot lifts from the floor and the limb advances forward; Mid Swing (MSw), the continuation of the limb advancement; Terminal Swing (TSw), the limb advancement is completed and prepares the position of the limb for stance.

Regarding what occurs during stance and swing, J. Perry in 1985 and J. Gage in 1991 [2] described the principal prerequisites of "normal gait" that are frequently lost in "pathological gait": a) Stability on stance: ability to maintain the center of mass (CoM) inside the base of support and provide balance, body propulsion and advancement of the limb in swing; b) Sufficient foot clearance during swing requires: appropriate position, power and stability of the ankle, knee and hip of the limb in stance phase; adequate ankle dorsiflexion, knee and hip dorsiflexion of the limb in swing; c) Appropriate prepositioning of the foot in terminal swing phase: still stability of the limb in stance phase, 
appropriate knee and proper foot position, adequate activity of dorsiflexors, inverters and everters of the foot; c) Adequate step length: adequate body balance, stable position in stance, adequate ankle dorsiflexion, hip and knee extension in swing; d) Energy conservation requires the capacity of minimizing the CoM excursion in all planes, adequate muscle forces, joint stability. In "pathological gait" impairments such as paresis, spasticity and muscle contractures can cause ambulation problems like instability during stance phase, balance disorders, limitation of foot clearance and require an increase in energy expenditure; therefore, the patient becomes unable to realize the prerequisites required and might produce "deviations" that can be finalized or not bypass the difficulties; sometimes "deviations" are really adaptative uses that profit by residual motor competence to maintain an independence in walking, other times "deviations" only cause problems for the patients. Some examples of adaptations of gait detectable during observational gait analysis [3] are: a) Limited ankle dorsiflexion at initial contact and stance which may be finalized to compensate impaired balance and provide stability at initial contact; b) Knee hyperextension of the affected side in stance might seek stability with the knee in extension; c) Diminished knee flexion of the affected side in swing could compensate the impaired balance in the desire to keep the foot close to the ground; d) Hip hiking or circumduction of the affected side and excessive ankle plantiflexion of the sound side in stance allows the clearance of the affected limb; e) Excessive hip and knee flexion in stance can compensate the muscle weakness with an adequate muscle length. Some "deviations" (like equinovarus foot or knee recurvatum) can cause pain, instability, risk of falling and an increase in energy costs; at times they are the only possible posture and so caution is required before removing them completely, often it is useful to correct these "deviations" in order to improve the patient's quality of life. To recognize a gait deviation and distinguish between a compensation or an obliged behaviour generated by a central nervous system (CNS) lesion, is probably the most important question to which we need answer in rehabilitation.

Thus the term "deviation" should not to be interpreted negatively when it becomes an advantageous solution for the neuromuscular system in order to realize forward progression, balance control and maintenance of support during walking. It is also important to identify the real causes of "deviations" to provide an understanding as to when their removal can actually be of help to the patient. For this purpose a combination of instrumental and clinical evaluation may be a valid tool to improve the diagnosis and determine which therapeutic intervention will correct the problems. That is why dynamic emg is largely combined with clinical assessment in patients with movement disorders after UM lesions.

Dynamic gait EMG is indeed a part of a rehabilitationprogram for patients with ambulation problems. The program usually includes: 1) the reason why the patient needs treatment, 2) goals of treatment, 3) time and modalities of intervention, 4) appropriate outcome measurement, 5) definition and verifying of the expected results.
The dynamic gait emg represents a necessary investigation to understand the clinical issue and provide a suitable answer to the patient's questions;

Dynamic emg recording reveals information on the timing and intensity of muscle activity during a movement. Caution is required in interpreting the intensity of the emg signal because the necessary comparison with the individual maximum voluntary activation is often compromised after CNS lesions. When dynamic emg during gait is used in terms of timing, it is reported in relation to a percentage of the gait cycle, which means the stance and the swing phases and their functional roles.

What we can observe is an alteration in timing of muscle activation (prolonged or curtailed) compared to the normal and /or a presence of coactivation of antagonist muscles. The literature $[1,2]$ provides ample description of normal and pathological gait emg patterns integrated with observational analysis, kinematic and kinetic data which may be used as reference. A good example is provided by the "stiff knee gait" pattern frequently observed in hemiplegic patients, which is a diminished and delayed peak knee flexion. The emg patterns associated with SKG is often an abnormal rectus femoris activity during the swing-phase, a time in which it doesn't normally work.

Another example is the varus foot, a deviation that can occur in stance and/or swing, associated or not with equinus; the muscles that are frequently the cause are tibialis anterior, tibialis posterior, flexor hallucis longus or flexor digitorum longus. Emg recording is a valuable tool for the identification of which muscle contributes to the varus and can help in choosing the correct surgical approach.

On completion of the instrumental examination a report should be written which covers the patient's problems, clinical evaluations, a description of emg features and suggestions.

Reports can be drawn up by filling in a standardized format or they may be a written description of signals made by the examiner.

We believe that a standardized format with several areas to fill-in at the beginning and at the end of our patient path evaluation, makes the production of reports easier and faster, allowing a more complete and comprehensible presentation of data, as well as improving the process of answering the patient's questions. Each laboratory usually produces a specific format that is based on the software used for the emg recording. Although reporting of the emg signals may take various forms it is essential that the examination modality and scales used are comprehensible and in accordance with the literature.

\section{LIST OF THE SEVERAL AREAS INCLUDED IN A REPORT}

1. Brief but precise description of the patient's history (History of Birth, Main Complaint, Past Treatments, Current Treatment)

\section{Observational gait analysis}

3. Description of the deformity analysed 
4. From the evaluation form, report only the items relevant to the deformity you have analysed and/or relevant for your treatment proposals

\section{List of recorded muscles}

6. Description of the EMG timing patterns

7. Conclusions leading to treatment proposals

8. Treatment proposals

\section{PATIENT'S HISTORY}

The Report should be comprehensive of all the necessary information for a good patient description.

In case of patients with cerebral palsy it is necessary to include accidents during birth which were responsible for the palsy; in case of adults, clinical information should be included regarding history prior to the examination.

The patient can require our intervention in different ways: the patient can be advised by the family, physician or physical therapist or voluntarily; in any case, it is important to know the reason why the patient come to us, how the ambulation difficulty is described by the patient, caregivers, physical therapist (i.e. need of assistance, foot dragging, falls...). It is very important to understand the main complaint, the current functional walking level at home, school, work and in the community, as well as other functional skills such as stair climbing (jumping and running for_children). Other essential information concerns: copresence of other clinically significant pathologies which may affect ambulatory ability; previous treatments (physical therapy, orthosis, walking aids, surgery, oral medication), specifying the type, when, how long (duration) and efficacy; focal intervention such as botulinum toxin o nerve blocks; the dosage, the muscles and nerves treated, the possible association with taping, serial casting and final results.

The same modality of description should be used with the current treatments specifying the reason why previous therapies were stopped or changed. Finally, in relation to the reason why the patient asked for our help, it is essential to know the patient or patient's family's expectations regarding the outcome of treatment, classified in order of importance.

\section{OBSERVATIONAL GAIT ANALYSIS}

Observational Gait Analysis (OGA) refers to a qualitative approach to gait analysis used by clinicians in which different ambulation behaviours or patterns are identified in patients through visual observations [4]. Every type of OGA consists of a checklist of gait "deviations" of single segments and joints observed during any single phase of gait cycle. Many forms have been developed to describe specific gait "deviations" in adults and children for neurological disorders, orthopaedic patients, amputees and rheumatoid arthritis patients. Krebs et al. [5] reported greater consistency among several videotape views of a patient walking than observing the subject directly during repeated walks. It has also been evidenced that slow motion videotaping markedly improves the consistency of observation. In order to identify the gait deviations correctly, Perry [1] suggested a form (developed by the Rancho Los Amigos Medical Center) with phases in which each gait deviation occurs; a further asset may be the differentiation between the most significant deviations and from others. As well as the Perry form, the Edinburgh Visual Gait Score (EVGE) is a good tool for cerebral palsy [6]; it presents 17 items: 6 for the foot, 4 for the knee, 2 for hip and pelvis, trunk, and clearance in swing; the score for each item is 0 (normal), 1 and 2 (deviate) with a total from 0 to 34 . The EVGE shows a good intra-observer and inter-observer reliability, and almost two-thirds of the observations correlated with instrumented 3D data, which means a good validity. These data were not fully confirmed in a recent study with inexperienced observers [7].

What is important is obtaining a form with a complete description of trunk, pelvis, hip, knee, foot and fingers positioning through gait cycle. As a clinical measure, a high level of accuracy and reliability in OGA measurements is essential to ensure optimal clinical practice; yet most OGA studies in the past provided evidence of reliability ranging from poor to moderate.

\section{DESCRIPTION OF THE DEFORMITY YOU HAVE ANALYSED}

The physical examination has six main goals: (1) to determine strength and selective motor control of isolated muscle groups; (2) to evaluate muscle tone and the influence of positional changes on tone ; (3) to estimate the degree of static deformity and/or muscle contracture at each joint; (4) to assess torsion and other deformities of the bone; (5) to describe fixed and mobile foot deformities; (6) to assess balance, equilibrium responses and standing posture [8]. The clinical examination allows us to identify which joints and muscles are impaired and to establish if those deformities may be passively corrected. Often gait laboratories develop a standard physical assessment form where it is possible to indicate the score of muscle strength, tone, range and selectivity of movement concerning hip, knee and ankle joints, and tests for biarticular muscles and bone deformities.

Muscle strength evaluation can be scored using the 6 levels (from 0 to 5) of the Kendall scale [9];

Selectivity of movements can be assessed by a 3 points scale of control movement in: 0 (no ability), 1 (partially isolated movement), 2 (complete selective movement) [8].

The Ashworth scale or modified Ashworth scale is commonly used for the clinical assessment of Spasticity, however, it is useful to consider its limits. These scales are designed to evaluate the resistance to a passive, velocitydependent stretch, but they have a low level of validity; i.e. point 4 of the scale describes the maximum level of resistance to a passive movement (no complete range of movement is possible) regardless of whether the cause is spasticity or contracture. The scale also has problems of reliability among different muscles groups (i.e. plantiflexors and knee extensors) [10]. It must be remembered that muscle tone can easily be influenced by the patient posture. Sometimes lower limb posture may cause confusion between spasticity and dystonia, both in children and adults. Examining the patient in supine position will not normally alter muscle tone in spastic patients but usually it will reveal a low tone in dystonic patients; furthermore, moving a dystonic rigid joint passively may produce a persistent resistance (similar to spasticity) or a gradual normalization of tone; spasticity will cause also an increase of deep tendon reflexes where dystonia will not [8]. Examination of muscle 
length using passive range of motion assessment helps to define a static joint restraint caused by shortness of antagonistic muscle group rather than spasticity or weakness. It is necessary to be aware of the interaction of multiple muscle groups at different joint levels. Some tests are useful to differentiate between biarticular and monoarticular muscle contracture. The Silfverskiold test is useful to differentiate tightness of the gastrocnemius and the soleus; the DuncanEly test provides information about rectus femoris spanning both the hip and the knee; the Phelps test assesses the role of biarticular adductor gracilis; the Thomas test is used to measure the degree of hip flexor tightness and the popliteal angle allow us to measure hamstring shift [8].

Bone deformity can play an important role in all varieties of cerebral palsy, thus it is useful to describe the presence of a limb length difference, femoral anteversion, patella alta, tibial torsion, forefoot and hindfoot position in weightbearing or not. A physical examination can help to correctly interpret emg data and to distinguish between structural - functional problems and compensation solutions; at the same time it must be borne in mind that this evaluation is static and, in part, a subjective examination.

\section{SELECTION OF THE MOST SIGNIFICANT IMPAIRMENT SIGNS}

In literature are described and classified gait patterns in spastic hemiplegia and spastic diplegia which can be a basis for a management algorithm.

Gait patterns in spastic hemiplegia in children and young adults were described in 1987 by Winter et al. [11] who distinguished four gait patterns: "drop foot" (group I), equinus only in swing phase mostly due to weakness of ankle dorsiflexors; "true equinus"(group II ) characterized by plantiflexion in stance phase caused by spasticity of triceps surae and associated or not to knee recurvatum; gastro-soleus spasticity or contracture plus a flexion "stiff knee gait" (group III); equinus, flexed stiff knee gait plus hip flexion and anterior pelvic tilt (group IV). Concerning spastic diplegia, Rodda et al. [12] refers about 4 other main patterns: true equinus, knee jump, apparent equinus, and crouch gait. For both types of patterns, hemiplegic and diplegic patients can show kinematic deviations of one or more lower limb joints and related muscle groups; the previous physical examination can help to identify a part of the prevalent muscle impairment: weakness, spasticity or contracture. However, it is often difficult to detect structural and functional problems with the static examination. As the authors described, the correct definition of a pattern and its causes can help us to choose an adequate therapeutic approach. In conclusion: according to the deformity selected during the clinical session it possible to produce a short summary of possible answers to the suggested questions (i.e. what are the main gait deviations?, what could the main causes be?, which muscles are involved?). Then a list of the muscles to be investigated with gait emg should be drawn up, with justification of choices made!

\section{LIST OF RECORDED MUSCLES AND EMG TIMING PATTERN}

Reports should usually contain a list of the muscles and recording modalities (i.e. types of electrodes used) and have a note about any difficulties that may occur. The principal factors influencing the surface emg (SEMG) signal, according to Farina et al. [13] are:

Intrinsic and functional properties of the motor units, anatomical features (thickness of the subcutaneous tissues), physical (amount of crosstalk from nearby muscles), the detection system (skin-electrode contact responsible for increased impedance and noise, spatial filter for signal detection, distance between electrodes, size and shape, inclination of the detection system relative to muscle fibre orientation, location of the electrodes over the muscle).

The method used to detect the stride phases (basography or optoelectronic systems) must be described. Different steps of assessment may be taken into consideration:

- $\quad$ Emg recording at rest (to exclude the presence of artefacts and verify the noise level of the electromyographer);

- $\quad$ Emg recording during fast and slow passive stretching of single muscle groups (the activity recorded may be significant of increased excitability to the stretch);

- $\quad$ Research of selective contraction of single muscles (to define the correct sites of electrodes and detect the presence of cross talk recorded from near muscles); this very useful test is difficult to perform in all children or adults with spastic paresis, however, this kind of research should be performed with more compliant patients.

- The patient can be asked to perform specific motor tasks requiring single joint movements (flexion and extension, repeated alternating movement, ...........).

- $\quad$ Finally emg gait recording will be performed with comfortable velocity or not. It is necessary to record several trials during the same session, at least a 10 meter walk.

\section{EMG TIMING PATTERN REPRESENTATION}

There is a great variety of representation of emg signals. Usually the electromyograph gives a first representation with a raw signal, then the emg trace may be rectified and filtered. An example of a single stride may be given in the Report, but it would be better to present more (3-5) consecutive strides of the same trial (Fig. 1a-c).

It is also possible to present an envelope of the signal (Fig. 2) (the number of the gait cycles required to form a representative profile of an average linear envelope varies between 3 [14] and six or ten [15].

The timing of emg activity related to the gait cycle is the only parameter which is helpful to describe the abnormal emg recording during walking, and which allows an interpretation to compare with normal gait.

J Perry [1] described seven different features of abnormal activity (Table 1a).

The presence of additional activity before the normal onset or beyond the normal off timing may represent a dynamic obstacle of the desired function or an appropriate support to an abnormal joint posture; a reduced activity 
TA

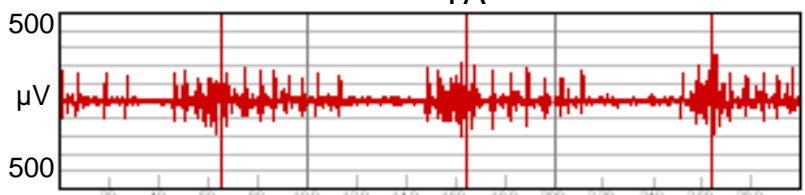

GCM

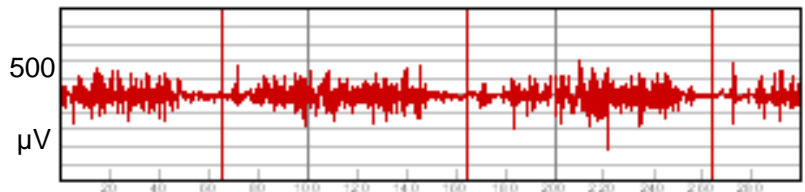

500

Fig. (1a). Raw emg signal representation of 3 consecutive stride.

TA

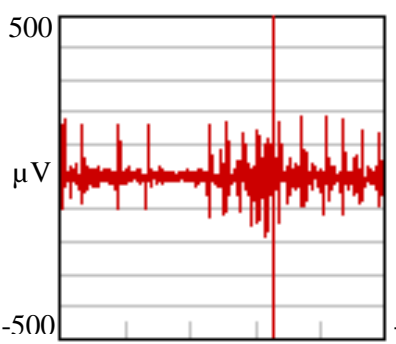

$\mathrm{SOL}$

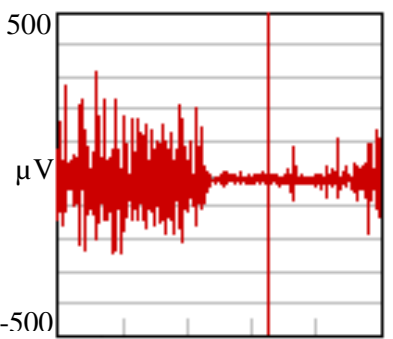

GCM

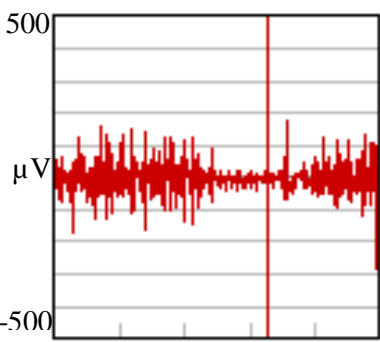

SOL

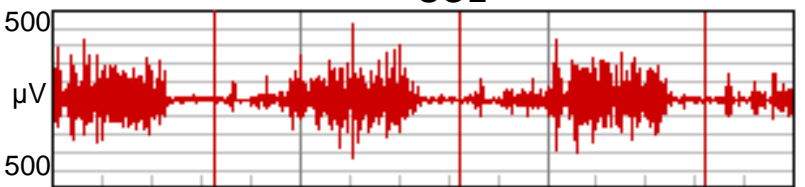

GCL

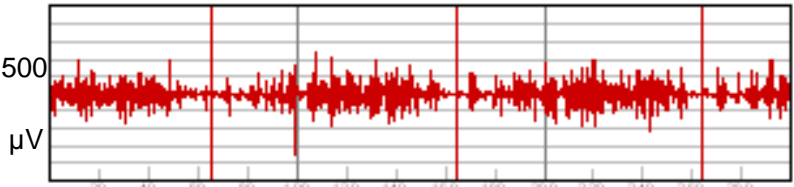

500

Fig. (1b). Raw emg signal representation of a single stride.
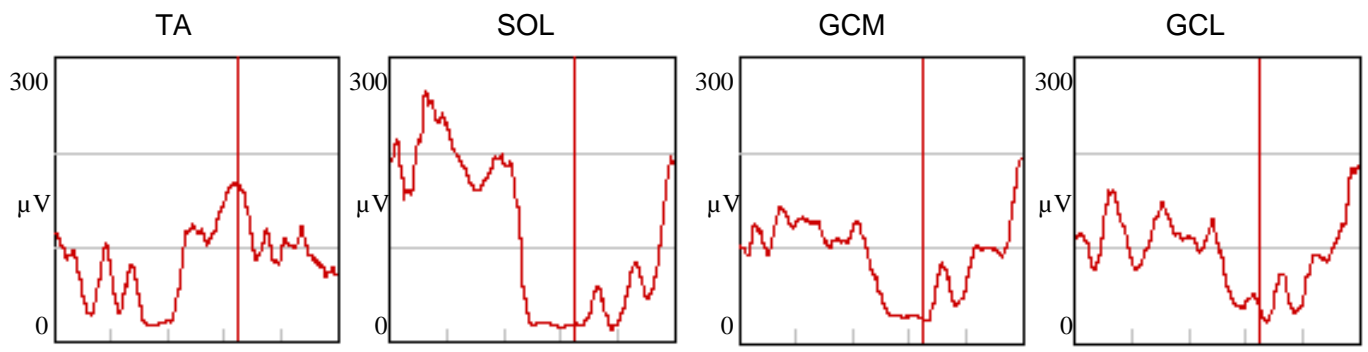

Fig. (1c). Emg signal of a single stride rectified and filtered.

(curtailed, delayed or absent) implies the lack of the desired activity; "continuous" activity is considered when the signal is uninterrupted for $90 \%$ or more of the gait cycle; "out of phase" activity in stance or swing can indicate a useful substitution activity.

The classification is very simple with few levels to define emg deviation, nevertheless it may be extended with other intermediate levels or further aspects (extra burst) which are not specified by J. Perry and Y. Blanc (Table 1b) added, to the list, the definition with both "premature and prolonged" as one feature of the signal aspects without it being considered continuous; the author also considers an "extra burst" activity like a period of Emg signal in addition to the main burst which can be normal or may belong to one of Perry's abnormal timing patterns. More than one extra burst can be observed. These "extra bursts" can be located at different times throughout the gait cycle: in stance, in swing and at the transition between stance to swing. Moreover "inversion of phase" is defined when the muscle is active in swing instead of stance, or muscle is active in stance instead of swing. The signal timing is the only useful information which helps the clinical process and requires the reference of normal SEMG timing and waveform. In the literature the description of Perry's studies may represent an adequate reference. To use the amplitude of the signal to this purpose can be misleading because of the possibility of interferences which are unrelated to the muscle activity, and for the same reason it is not advisable to compare the different amplitudes of different muscles.

To complete the report, it is important to take into account other factors such as walking velocity, acceleration or deceleration effects and fatigue.

\section{CONCLUSIONS LEADING TO TREATMENT PROPOSALS}

In order to use the emg results for choosing different treatment programs, the description of the timing pattern must lead to the identification of peripheral and central 
mechanisms (contracture, paretic, spastic, co-contraction components), which can significantly interfere with the gait profile of individual patients.

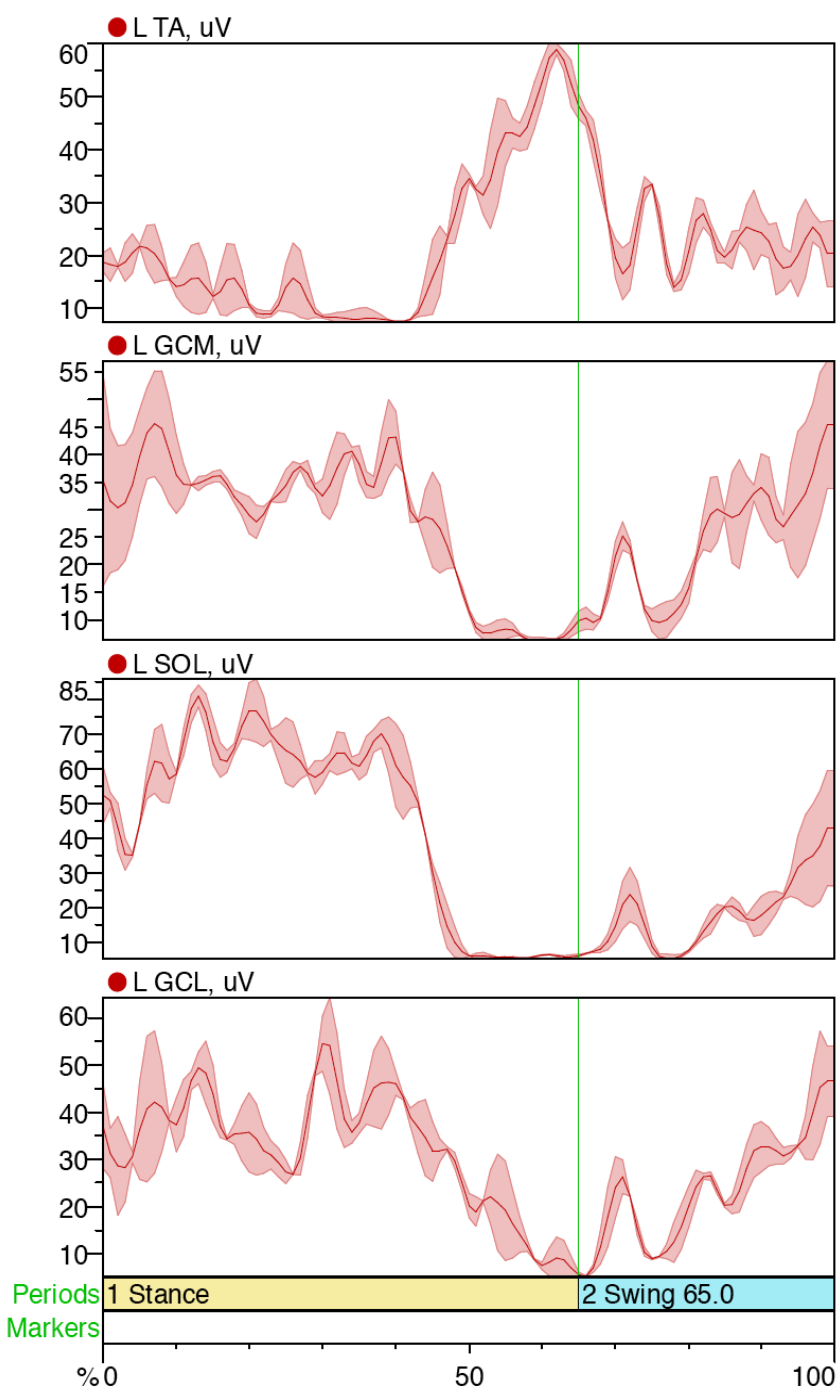

Fig. (2). Average linear envelope of 3 gait cycles represented in Fig. (1).
As recently reported by Frigo and Crenna [16] the principal mechanisms include:

a) Changes in the passive muscle-tendon properties (peripheral non neural component).

b) Defective activation or modulation of motor unit recruitment (paretic component).

c) Abnormal activity profile during muscle lengthening (spastic component).

d) Loss of selective activation with simultaneous activation of functionally antagonist muscles (cocontraction).

Table 1a. Different Features of Abnormal emg Activity Related to the Gait Cycle from: Perry J. Gait Analysis: Normal and Pathological Function. Slack 1992)

\begin{tabular}{|c|c|}
\hline \multicolumn{2}{|c|}{ Emg Timing Deviation (J Perry 1992) } \\
\hline Deviation & Definition \\
\hline \hline Premature & Action begins before the normal onset \\
\hline Prolonged & Action continues beyond the normal cessation time \\
\hline Continuous & Emg uninterrupted for 90\% or more of the gait cycle \\
\hline Curtailed & Early termination of the Emg \\
\hline Delayed & Onset later than normal \\
\hline Absent & Emg of insufficient amplitude or duration \\
\hline Out of Phase & Swing or stance time reversed \\
\hline
\end{tabular}

Changes in rheological properties of muscle-tendon systems can be characterized by absence of Emg signal; reduced or absent Emg activity observed during isolated voluntary contractions does not allow reliable predictions on defective activation of the same muscles during walking. In fact SEMG signals do not provide any good estimate of muscle force, the more so under dynamic conditions (e.g. locomotor movements). SEMG recording during walking can also be used for objective detection of the possible interference of a spastic component, i.e. of abnormally increased EMG recruitment during muscle lengthening

Table 1b. Extension of Emg Timing Deviation Proposed by Y. Blanc (1996)

\begin{tabular}{|c|c|}
\hline \multicolumn{2}{|c|}{ Emg Timing Deviation (J Perry 1992 Modified by Y Blanc) } \\
\hline Deviation & Definition \\
\hline \multicolumn{2}{|l|}{ Normal } \\
\hline Absent & No Emg detected (insufficient amplitude or duration) \\
\hline Premature & Action begins before the normal onset \\
\hline Prolonged & Action continues beyond the normal cessation time \\
\hline Premature and Prolonged & Signal aspects without that it may be considered continuous \\
\hline Continuous & Emg uninterrupted for $90 \%$ or more of the gait cycle \\
\hline Curtailed & Early termination of the Emg \\
\hline Inversion of Phase & Muscle is active in swing instead of stance or muscle is active in stance instead of swing \\
\hline Extra burst in Stance, in Swing or transition Stance/Swing & $\begin{array}{l}\text { Emg activity in addition of the main burst which can be normal or belongs to an abnormal } \\
\text { timing pattern. More than one extraburst can be observed }\end{array}$ \\
\hline
\end{tabular}


phases occurring throughout the stride cycle [17]. The correlation between SEMG and kinematic can provide clues for the identification of dynamic spastic phenomena. Gait disturbances are frequently associated with poorly selective activation of functionally antagonist muscles, resulting in increased co-contraction levels. It can match with the "out of phase" described by Perry or the "extra bursts". In this case antagonistic muscles simultaneously attain recruitment levels higher than a pre-determined noise threshold influencing the

agonists activity. JM Gracies [18] described this phenomenon as "spastic co-contraction", where the agonist activity (i.e. foot dorsiflexors) is delayed and not sufficient to counterbalance the antagonists strength (i.e. foot plantarflexors).

The description of emg signals must, in a adequate way to OGA and physical examination, help us to understand if the activity of some evaluated muscles can be an explanation for the "pathologic deviation" we think have to be modified.

Furthermore the emg results can lead also to the identification of the single mechanisms (contracture, paresis, spasticity, co-contraction) where it is necessary to interfere with the treatments.

Indeed we are not always able to define with the Emg recording the indications for treatment, sometime we need to use other evaluations like a study of kinematic, kinetic or a diagnostic nerve block to confirm our diagnostic hypothesis and make a choice

\section{TREATMENT PROPOSALS}

After the list on muscles, the description of their timing in association or not with other additional necessary tasks, the description of the relation between deviations and emg activity is important to indicate the possible therapeutic proposals:

1. No treatment but regular observation through clinical and gait analysis

2. Physical Therapy

3. Orthosis

4. Walking Aids

5. Pharmacological Treatment (Oral drugs, Botulinum Toxin, Phenol nerve block)

6. Neurosurgery

7. Orthopaedic surgery

\section{CONCLUSION}

In conclusion, the report should reflect the decisionmaking process by which the clinical evaluation and the instrumental analysis of gait dynamic emg have led to a diagnosis and to the planning of related therapeutic solutions. The Report may include forms and tables besides the reproduction of emg signals. Regarding physical examination, observational gait analysis and emg pattern classification and recording parameter attention must be done for an adequate support of literature.

\section{REFERENCES}

[1] Perry J. Gait Analysis: Normal and pathological function. USA: Slack 1992.

[2] Gage JR. Gait Analysis in cerebral palsy. London: Mac Keith Press 1991; 61-95

[3] Olney SJ. Richards C. Hemiparetic gait following stroke. Part I: Characteristics. Gait Posture 1996; 4: 136-48.

[4] Malouin F. Observational gait analysis. In: Craik RL, Oatis CA, Eds. Gait Analysis: Theory and Applications. Mosby 1995; pp. 112-24.

[5] Krebs DE, Edelstein JE, Fishman S. Reliability of observational kinematic gait analysis. Phys Therapy 1985; 65: 1027-33.

[6] Read HS, Hazlewood ME, Hillman SJ, Prescott RJ, Robb JE. Edinburgh visual gait score for use in cerebral palsy. J Pediatr Orthop 2003; 23: 296-301

[7] Ong OML, Hillman SJ, Robb JE. Reliability and validity of the Edinburgh Visual Gait Score for cerebral palsy when used by inexperienced observers. Gait Posture 2008; 28: 323-6.

[8] Trost J. Physical assessment and observational gait analysis. In: JR Gage, Ed. The treatment of gait problems in cerebral palsy. London: Mc Keith Press 2004; 71-89.

[9] Kendall HO, Kendall FP, Wadsworth GE. Muscle testing and function. Baltimore, Maryland: Wiliams and Wilkins, USA 1971; $1-22$.

[10] Pandyan AD, Johnson GR, Price CIM, Curless RH, Barnes MP, Rodgers H. A review of properties and limitations of the Ashworth and modified Ashworth Scales as measures of spasticity. Clin Rehabil 1999; 13: 373-83.

[11] Winters TF, Gage JR. Gait patterns in spastic hemiplegia in children and young adults. J Bone Joint Surg Am 1987; 69(3): 43741.

[12] Rodda JM, Graham HK, Carson L, Galea MP, Wolfe R. Sagittal gait patterns in spastic diplegia. J Bone Joint Surg Br 2004; 86(2): 251-8.

[13] Farina D, Merletti R, Enoka RM. The extraction of neural strategies from the surface EMG. J Appl Physiol 2004; 96(4): 1486-95.

[14] Arsenault AB, Winter DA, Marteniuk RG, Hayes KC. How many strides are required for the analysis of electromyographic data in gait? Scand J Rehabil Med 1986; 18: 133-5.

[15] Shiavi R, Frigo C, Pedotti A. Electromyographic signals during gait: criteria for envelope filtering and number of strides. Med Biol Eng Comput 1998; 36(2): 171-8.

[16] Frigo C, Crenna P. Multichannel SEMG in clinical gait analysis: A review and state-of-the-art. Clin Biomech 2009; 24(3): 236-45.

[17] Crenna, P. Spasticity and spastic gait in children with cerebral palsy. Neurosci Biobehav Rev 1998; 22: 571-8.

[18] Gracies JM. Pathophysiology of spastic paresis II: emergence of muscle overactivity. Muscle Nerve 2005; 31(5): 552-71. 